\title{
Synchronous Digitization for Modulated Signal Analysis
}

\author{
Ryan Muir, Shane Sullivan, Robert Oglesbee, and Garth Simpson
}

Purdue University, West Lafayette, IN USA

The signal to noise $(\mathrm{S} / \mathrm{N})$ of a nonlinear optical measurement is one of the critical defining properties dictating the available scope of use of emerging nonlinear optical microscopy techniques. Several strategies have been adapted to optimize $\mathrm{S} / \mathrm{N}$ in nonlinear imaging systems. In regard to recovering the signal measured by the optical sensor, two general measurement strategies have emerged for obtaining high $\mathrm{S} / \mathrm{N}$ images, depending on the intensity of the observed signal (Figure 1). Threshold discrimination photon counters are often used for trace signal detection, while gated integration and lock-in amplification (LIA) are more common for measuring higher intensity signals. LIA is particularly suited for modulated imaging systems and high speed data acquisition.

In a previous publication[1], we presented the synchronous digitization (SD) signal recovery technique, in which the voltage peak of every photomultiplier tube (PMT) signal transient was flash analog-todigital-converted (ADC) at a rate of $80 \mathrm{MHz}$ in a second harmonic generation (SHG) microscope. Direct computer access to every digitized sample enabled a unique analysis in which we simultaneously counted and integrated in software, and the result which provided the highest $\mathrm{S} / \mathrm{N}$ was selectively saved. By combining and scaling both datasets, $\mathrm{S} / \mathrm{N}$ was recovered at $87 \%$ to approaching $100 \%$ of the Poisson $\mathrm{S} / \mathrm{N}$ limit across the entire dynamic range of the PMT, and allowed SD to span the entire scope of space in Figure 1.

For modulation-based imaging techniques, the depth of modulation (DoM) is a common measure of the relative modulation amplitude, defined by the modulation peak amplitude divided by the mean signal value. The depth of attenuation (DoA) is an alternative measure of the relative modulation amplitude and is defined as the peak-to-peak modulation amplitude divided by the peak modulation amplitude. DoA is a very simple data analysis technique which can recover $\mathrm{S} / \mathrm{N}$ much greater than the absolute measure of modulation amplitude (Figure 2). Intriguingly, the $\mathrm{S} / \mathrm{N}$ from DoA can exceed the Poisson $\mathrm{S} / \mathrm{N}$ limit by many times at high modulation amplitudes.

A conventional LIA and SD-DoA S/N comparison was performed. A polarization-modulated, $1060 \mathrm{~nm}$ $\mathrm{Nd}$ :YAG laser beam was directed into an SHG microscope, and SHG signal from a z-cut quartz slide was collected in the return direction by a PMT. Data was either flash digitized synchronously with the laser by computer data acquisition cards, or measured by a LIA. The $\mathrm{S} / \mathrm{N}$ is plotted against the incident laser power in Figure 3 for both SD-DoA and LIA. The time constant of the LIA was set to $100 \mu \mathrm{s}$, where five time constants of integration time are required for the LIA to reach $99 \%$ of its final value. Even under near ideal conditions for the LIA as described in Figure 1, $500 \mu \mathrm{s}$ SD-DoA provides nearly double the $\mathrm{S} / \mathrm{N}$ compared to LIA. Recording five times less data with $100 \mu \mathrm{s} \mathrm{SD}-\mathrm{DoA}$ returns a $\mathrm{S} / \mathrm{N}$ approximately on par with the capabilities of LIA. Compared to software emulation of the LIA with the digitized data, SD-DoA provided a $\mathrm{S} / \mathrm{N}$ improvement factor of $\gamma \approx 1.6$ for all measured points of $500 \mu \mathrm{s}$ SD-DoA. This is consistent with the expected improvement for the experimentally measured modulation amplitude of DoA $=60 \%$ from Figure 2. Even higher improvement factors are observed compared to the conventional LIA, owing to the inherent inefficiency of RC integration, as well as the poor performance of integration methods in the low signal limit. 


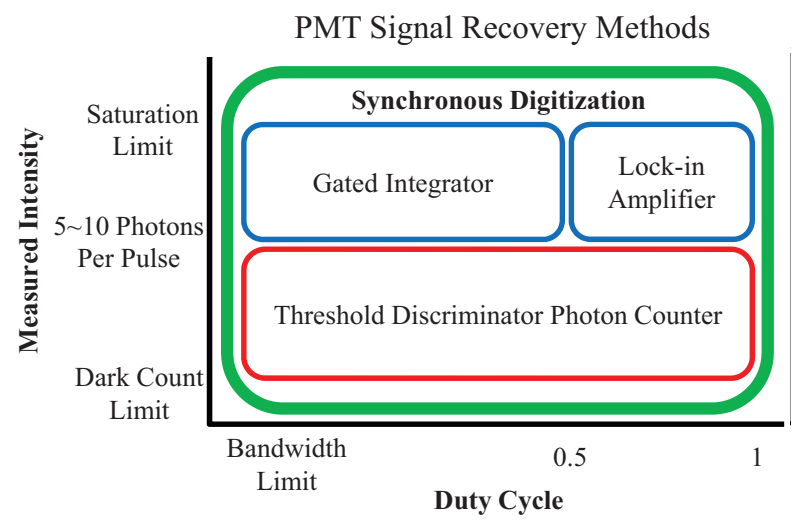

Fig. 1. Adapted from a Stanford Research Systems application note[2], a visual representation of the main signal recovery methods and the conditions under which they are optimized. With $\mathrm{SD}, \mathrm{S} / \mathrm{N}$ can be maximized across the scope of space of duty cycle and signal intensity, indicated by the outer green box.

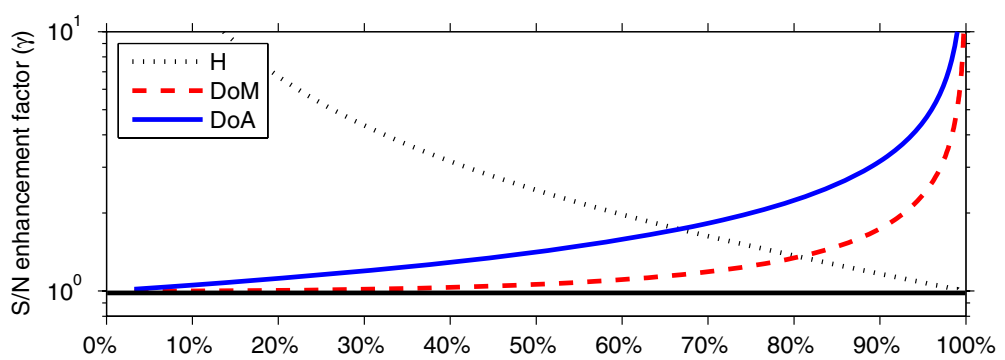

Fig. 2. The $\mathrm{S} / \mathrm{N}$ enhancement factor, $\gamma$, of the DoM and DoA analytical methods, relative to the shotnoise limited $\mathrm{S} / \mathrm{N}$ of the absolute modulation amplitude. DoA in particular returns a $\mathrm{S} / \mathrm{N}$ much higher than the absolute modulation amplitude, and can even exceed the Poisson $\mathrm{S} / \mathrm{N}$ limit (shown as line $\mathrm{H}$ ) for DoA amplitudes greater than $70 \%$.

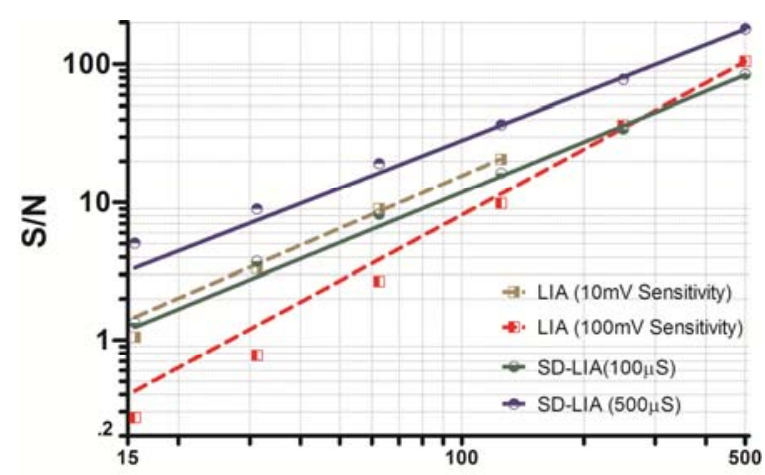

Incident Laser Power (mW)

Fig. 3. S/N comparison of SD-LIA with DoA data processing and conventional LIA as a function of incident laser power along with linear fits of the data points. Two different sensitivity ranges were used on the LIA to span the wide dynamic range with high $\mathrm{S} / \mathrm{N}$. Even under ideal conditions for LIA, $500 \mu \mathrm{s}$ SD-LIA with DoA analysis provided nearly twice the $\mathrm{S} / \mathrm{N}$ compared to conventional LIA.

1. Muir, R.D., D.J. Kissick, and G.J. Simpson, Optics Express 20(2012), p. 10406.

2. (2004) Stanford Research Systems, Signal Recovery with PMTs.

3. The authors gratefully acknowledge support from the NIH Grant Number R01GM-103401 and support from Eli Lilly and Co. from the Research Awards Program. 